\title{
Impaired chronotropic response to physical activities in heart failure patients
}

\author{
Hong Shen ${ }^{1 \dagger}$, Jianrong Zhao ${ }^{2 \dagger}$, Xiaohong Zhou ${ }^{3}$, Jingbo $\mathrm{Li}^{i^{*}}{ }^{\mathbb{D}}$, Qing Wan ${ }^{1}$, Jing Huang ${ }^{1}$, Hui Li ${ }^{2}$, Liqun Wu ${ }^{4}$, \\ Shungang Yang ${ }^{3,5}$ and Ping Wang 3,5
}

\begin{abstract}
Background: While exercise-based cardiac rehabilitation has a beneficial effect on heart failure hospitalization and mortality, it is limited by the presence of chronotropic incompetence (Cl) in some patients. This study explored the feasibility of using wearable devices to assess impaired chronotropic response in heart failure patients.

Methods: Forty patients with heart failure (left ventricular ejection fraction, LVEF: $44.6 \pm$ 5.8; age: $54.4 \pm 11.7$ ) received ECG Holter and accelerometer to monitor heart rate $(\mathrm{HR})$ and physical activities during symptom-limited treadmill exercise testing, 6-min hall walk (6MHW), and 24-h daily living. Cl was defined as maximal HR during peak exercise testing failing to reach 70\% of age-predicted maximal HR (APMHR, 220 - age). The correlation between HR and physical activities in Holter-accelerometer recording was analyzed.

Results: Of 40 enrolled patients, 26 were able to perform treadmill exercise testing. Based on exercise test reports, 13 (50\%) of 26 patients did not achieve at least 70\% of APMHR (Cl patients). Cl patients achieved a lower \% APMHR $(62.0 \pm 6.3 \%)$ than non-Cl patients who achieved $72.0 \pm 1.2 \%$ of APMHR $(P<0.0001)$. When Holter-accelerometer recording was used to assess chronotropic response, the percent APMHR achieved during 6MHW and physical activities was significantly lower in $\mathrm{Cl}$ patients than in non-Cl patients. Cl patients had a significantly shorter 6MHW distance and less physical activity intensity than non-Cl patients.

Conclusion: The study found impaired chronotropic response in 50\% of heart failure patients who took treadmill exercise testing. The wearable Holter-accelerometer recording could help to identify impaired chronotropic response to physical activities in heart failure patients.
\end{abstract}

Trial registration: ClinicalTrials.gov ID NCT02358603. Registered 16 May 2014.

Keywords: Chronotropic incompetence, Heart rate, Heart failure rehabilitation, Treadmill exercise testing, 6 min hall walk

\section{Background}

Heart failure patients experience a variety of symptoms, of which the most frequently presenting symptoms are exertional breathlessness, fatigue, and intolerance to physical activities, leading to poor quality of life. While, historically, patients with heart failure were suggested to avoid exertion, exercise-based cardiac rehabilitation has now been recognized as having a beneficial effect on heart failure hospitalization and mortality [1-4]. Currently, exercise prescription is commonly based on

\footnotetext{
* Correspondence: jbli@medmail.com.cn

${ }^{\dagger}$ Equal contributors

'Department of Cardiology, Shanghai Sixth People's Hospital, Shanghai Jiao

Tong University, Shanghai, China

Full list of author information is available at the end of the article
}

heart rate. However, exercise level or target intensity that depends on achievable maximal heart rate can be significantly limited in heart failure patients with chronotropic incompetence (CI) [3, 4].

In response to physical exercise that increases oxygen demand, heart rate (HR), via enhanced sympathetic activity and/or withdrawal of parasympathetic activity, increases to meet the body's metabolic demand. $\mathrm{CI}$ is a condition when HR increases inadequately in response to increased metabolic demand [5-8]. Patients with CI often show exercise intolerance and hence have impaired quality of life. Furthermore, studies have also demonstrated that $\mathrm{CI}$ is an independent risk factor for major cardiovascular adverse events including overall mortality [9-12]. The 
prevalence of $\mathrm{CI}$ in patients with heart failure has been reported to vary from $25 \%$ to $65 \%[8,9,13,14]$.

At present, the method that clinically assesses CI is dynamic incremental exercise testing or exercise tolerance testing during which cardiac chronotropic capacity is measured [6-12]. CI is believed to be present if maximal HR during the peak of exercise testing cannot reach a certain percentage of the age-predicted maximal heart rate value (APMHR, usually 220 - age), such as $80 \%$, or even $70 \%[6-8,12]$. The magnitude of the change in $\mathrm{HR}$ during exercise testing is also used to detect impaired chronotropic incompetence, e.g., the HR reserve (the difference between maximal HR during peak exercise and resting HR prior to exercise testing) $[8,9,11,12]$.

While exercise-based cardiac rehabilitation has been recommended for heart failure patients, the adoption of the guidelines is negatively impacted by CI. In other words, if $\mathrm{CI}$ is assessed and better managed, target intensity and exercise formula can be better prescribed. The purpose of the present study was to investigate a method of heart rate and physical activity recording to assess chronotropic response during treadmill exercise testing and daily physical activities in patients with heart failure.

\section{Methods}

The study was conducted in two centers and the study protocol was approved by both hospitals' Institutional Review Boards and in compliance with the Declaration of Helsinki. All patients completed written informed consent.

\section{Selection of patients}

Forty patients were enrolled between June 2014 and April 2015. The inclusion criteria included: (1) patients had heart failure based on ESC 2012 heart failure guidelines; (2) patients' NYHA classification was ranged from I to III and left ventricular ejection fraction (LVEF) was more than 35\%; (3) patients were capable of performing moderate exercise. Patients were excluded if they (1) received a pacemaker, implantable cardioverter-defibrillator or cardiac resynchronization therapy device; (2) had persistent or permanent atrial fibrillation; (3) were incapable of exercise due to angina, heart failure decompensation, ST deviation $>2 \mathrm{~mm}$, or active pericarditis and myocarditis; (4) had acute myocardial infarction $<45$ days; (5) had uncontrolled hypertension; or (6) had other medical issues that would compound the present study.

\section{Study procedures}

All enrolled patients received 24-h Holter ECG (NorthEast Monitoring, Inc., Maynard, MA, USA) for HR and ActiGraph GT3 accelerometer (ActiGraph, Shalimer, FL, USA) monitoring for determining the magnitude of physical activities. The clinically validated Actigraph accelerometer determined counts of 10-s acceleration activities in $\mathrm{X}-, \mathrm{Y}-$, and $\mathrm{Z}$ - vectors with each count equal to $16 \mathrm{~m}-\mathrm{g}$ per second where the $\mathrm{g}$ is $9.825 \mathrm{~m} \cdot \mathrm{s}^{-2}$ [15-17]. The vector magnitude (VM) was calculated as the square root of the sum of the second power of X-, Y-, and Z-vector counts. Once the Holter-Actigraph monitoring was in place, patients underwent a 6 min hall walk $(6 \mathrm{MHW})$ and a symptom-limited treadmill exercise test. Some patients, based on physicians' clinical judgment and patients' willingness, did not participate in treadmill exercise testing. All enrolled patients received stable medication and there was no requirement to withhold any medication. Patients' demographic information, echocardiography, blood pro-NT-BNP, and medications were collected at enrollment.

The symptom-limited maximal treadmill exercise test adopted the modified Naughton-Balke method [18]. Briefly, the total time of treadmill exercise testing was 14 min with two minutes at base speed with slope 0 (no inclination), two minutes at slope 1 level and speed 2, and two minutes at each of next five slopes with no change in speed. During exercise testing, 12-lead electrocardiogram and blood pressure were recorded in addition to Holter-Actigraph recording.

\section{Assessment of chronotropic incompetence}

The assessment of chronotropic incompetence was based on the achieved maximal HR during peak treadmill exercise. $\mathrm{CI}$ is defined as maximal $\mathrm{HR}$ at peak treadmill exercise that fails to reach an arbitrary percentage of APMHR, for which $70 \%$ was chosen as the criterion for diagnosis of $\mathrm{CI}$ in heart failure patients $[12,19,20]$. In the present study, as most patients took beta-blockers, a newly proposed equation based on HR reserve for determining chronotropic impairment was also used to assess CI, e.g., chronotropic index for patients with beta blockers (called chronotropic index $-\beta)=(\mathrm{HR}$ at peak exercise - resting HR) / (119 + (resting HR / 2) - (age / 2) - resting HR) [11].

Chronotropic response during daily living was assessed by 24-h Holter-Actigraph recording. An event of physical activity was defined once Actigraph recording reported 10 or more than $10 \mathrm{~s}$ of activities. Maximal Actigraph VM value in counts and HR were determined for each physical activity. Resting HR was determined by averaging HR values in the time window from $10 \mathrm{~min}$ after the previous activity event to the time right before the incoming activity. Thus, for each physical activity, three parameters, e.g., resting HR, maximal HR and maximal Actigraph VM, were obtained. The regression between HR values and corresponding Actigraph activity levels was performed for 24-h daily living. The overall intensity of daily physical activities was expressed as the averaged maximal VM of all physical activities during the recording window. 


\section{Statistical analysis}

Continuous variables are expressed with mean \pm standard deviation. The categorical variables are used for number of patients. Comparisons for continuous variables were made using $t$ test whenever appropriate. Categorical variables were compared using the Chi square test. The correlation between the $\mathrm{CI}$ values by exercise test reports and those by Holter-Actigraph recording was determined using Pearson correlation coefficients with corresponding correlation equations. The simple linear regression between HR and physical activities for 24-h Holter-Actigraph recording was performed. To minimize the impact of variations among individuals on regression analysis results, HR was taken as a function of the percentage of the maximal daily physical activity. Binary logistic regression was used to detect the effect of variables in patient characteristics (age, weight, gender, coronary heart disease, cardiomyopathy, LVEF, NYHA, $\beta$-blockers) on the results (CI or no-CI) of treadmill exercise testing. A twotailed $P$ value of $\leq 0.05$ was considered significant. The statistical tool SPSS was used for the statistical analyses.

\section{Results}

Treadmill exercise testing

All 40 enrolled patients (median age: 57.5 years old, range 30-70) were diagnosed with heart dysfunction with LVEF $44.6 \pm 5.8 \%$ and symptoms of exertionassociated dyspnea, asthenia and fatigue. Most patients (60\%) were in NYHA class II. The clinical characteristics of patients are presented in Table 1 . Of the 40 enrolled patients, 26 patients underwent treadmill exercise testing and the remaining 14 patients declined treadmill exercise testing. There was no significant difference in clinical characteristics between patients taking treadmill exercise testing and those not taking (Table 2). In 13 of 26 patients (50\%) who underwent treadmill exercise testing, maximal heart rate at peak exercise did not reach at least $70 \%$ of APMHR (CI patients) while in the remaining 13 patients (50\%) maximal heart rate did reach $\geq 70 \%$ of APMHR (non-CI patients). Of 5 patients with NYHA III, maximal heart rate in 2 patients reached $\geq 70 \%$ of APMHR but not in other 3 patients. The binary logistic regression analysis showed only gender affected the CI results of treadmill exercise testing with an odd ratio 0.04 (for male), $0.002-0.80595 \% \mathrm{CI}$ and $P$ value $=0.036$.

Table 1 Patient characteristics and results of treadmill exercise testing

\begin{tabular}{|c|c|c|c|c|}
\hline & All patients $(N=40)$ & $\geq 70 \%$ APMHR $(N=13)$ & $<70 \%$ APMHR $(N=13)$ & $P$ value \\
\hline Age (years) & $54.4 \pm 11.7$ & $53.1 \pm 10.9$ & $52.5 \pm 13.8$ & 0.905 \\
\hline Weight (Kg) & $74.8 \pm 17.6$ & $78.2 \pm 19.3$ & $74.4 \pm 19.4$ & 0.634 \\
\hline Male, N (\%) & $30(75)$ & $13(92.3)$ & $7(53.8)$ & 0.005 \\
\hline NYHA class & $2.2 \pm 0.6$ & $1.9 \pm 0.6$ & $2.2 \pm 0.4$ & 0.166 \\
\hline LVEF (\%) & $44.6 \pm 5.8$ & $45.8 \pm 5.5$ & $42.0 \pm 5.2$ & 0.090 \\
\hline $\operatorname{LVESD}(\mathrm{mm})$ & $45.9 \pm 7.6$ & $45.2 \pm 7.1$ & $49.9 \pm 4.8$ & 0.066 \\
\hline LVEDD (mm) & $59.4 \pm 7.7$ & $58.2 \pm 7.8$ & $62.8 \pm 4.5$ & 0.084 \\
\hline $6 \mathrm{MHW}(\mathrm{m})$ & $413.8 \pm 86.9$ & $486.2 \pm 62.0$ & $416.7 \pm 37.1$ & 0.003 \\
\hline $\mathrm{SBP}(\mathrm{mmHg})$ & $125.6 \pm 18.1$ & $126.6 \pm 20.1$ & $126.8 \pm 17.4$ & 0.987 \\
\hline $\mathrm{DBP}(\mathrm{mmHg})$ & $76.8 \pm 9.9$ & $79.2 \pm 10.0$ & $76.2 \pm 11.4$ & 0.528 \\
\hline BNP (pg/mL) & $147.5 \pm 225.8$ & $114.7 \pm 96.8$ & $124.0 \pm 145.9$ & 0.855 \\
\hline$C A D, N(\%)$ & $15(37.5)$ & $4(33.3)$ & $5(35.7)$ & 0.680 \\
\hline Cardiomyopathy, N (\%) & $35(87.5)$ & $11(84.6)$ & $12(92.3)$ & 0.539 \\
\hline B-blocker, N (\%) & $38(95.0)$ & $12(92.3)$ & $13(100.0)$ & 0.308 \\
\hline ACE inhibitor, N (\%) & $16(40.0)$ & $6(46.2)$ & $8(61.5)$ & 0.431 \\
\hline Diuretics, N (\%) & $20(50.0)$ & $7(53.8)$ & $7(53.8)$ & 1.000 \\
\hline Digoxin, N (\%) & $10(25.0)$ & $2(15.4)$ & $6(46.2)$ & 0.089 \\
\hline \multicolumn{5}{|c|}{ Patients taking treadmill exercise testing $(N=26)$} \\
\hline Rest HR (bpm) & $78.2 \pm 10.9$ & $80.1 \pm 10.1$ & $76.2 \pm 11.8$ & 0.373 \\
\hline Maximum HR (bpm) & $112.0 \pm 14.8$ & $120.1 \pm 8.8$ & $103.9 \pm 15.4$ & 0.003 \\
\hline HR reserve (bpm) & $33.9 \pm 11.9$ & $40.0 \pm 10.5$ & $27.2 \pm 10.2$ & 0.006 \\
\hline APMHR & $167.6 \pm 12.5$ & $166.8 \pm 11.4$ & $168.4 \pm 14.0$ & 0.775 \\
\hline Achieved \%APMHR & $67.0 \pm 6.8$ & $72.0 \pm 1.2$ & $62.0 \pm 6.3$ & $<0.0001$ \\
\hline$C$ index- $\beta(\%)$ & $62.7 \pm 19.6$ & $75.3 \pm 12.9$ & $46.4 \pm 15.3$ & $<0.0001$ \\
\hline
\end{tabular}


Table 2 Baseline characteristics in patients with or without treadmill exercise testing

\begin{tabular}{llll}
\hline & TET $(N=26)$ & No TET $(N=14)$ & $P$ value \\
\hline Age (years) & $52.8 \pm 12.4$ & $57.5 \pm 9.4$ & 0.233 \\
Weight (Kg) & $76.3 \pm 19.4$ & $72.0 \pm 13.0$ & 0.471 \\
Male, N (\%) & $20(76.9)$ & $10(71.4)$ & 0.702 \\
LVEF (\%) & $43.9 \pm 5.7$ & $45.8 \pm 5.9$ & 0.346 \\
NYHA class & $2.1 \pm 0.5$ & $2.3 \pm 0.7$ & 0.318 \\
LVESD (mm) & $47.5 \pm 6.5$ & $42.8 \pm 8.5$ & 0.062 \\
LVEDD (mm) & $60.5 \pm 6.8$ & $57.2 \pm 8.8$ & 0.208 \\
6MHW (m) & $451.4 \pm 61.8$ & $344.1 \pm 83.9$ & $<0.001$ \\
BNP (pg/mL) & $119.4 \pm 123.8$ & $119.8 \pm 336.1$ & 0.294 \\
CAD, N (\%) & $9(34.6)$ & $5(35.7)$ & 0.945 \\
Cardiomyopathy, N (\%) & $23(88.5)$ & $12(85.7)$ & 0.802 \\
B-blocker, N (\%) & $25(96.2 \%)$ & $13(92.9)$ & 0.648 \\
\hline
\end{tabular}

TET treadmill exercise testing

When $\beta$-blocker use was taken into account in the analysis using chronotropic index- $\beta, 38.5 \%$ patients (10/26) failed to achieve $60 \%$ chronotropic index- $\beta$. While there were no significant differences in age and corresponding APMHR between $\mathrm{CI}$ and non-CI patients, CI patients achieved significantly lower maximal HR, HR reserve, and chronotropic index- $\beta$ than non-CI patients (Table 1).

Figure 1a shows an example of HR and physical activity levels recorded by the Holter-Actigraph devices during treadmill exercise testing in a patient. There was a significant correlation between HR and activity levels, e.g., $\mathrm{HR}=(0.0629 \times \mathrm{VM})+80.999(R=0.7322$, $P<0.0001$, Fig. 1b), demonstrating an increase in HR in response to the increase in physical activity levels. The magnitude of physical activity level (VM) during peak exercise appeared lower in CI patients (VM: $380.3 \pm 194.5$ unit counts) than in non-CI patients (VM: $475.5 \pm 142.0$ unit counts, $P=0.183$ vs. the $C I$ patients).

\section{HR analysis during 24-h daily living}

An example of the relationship between HR and the VM of physical activities from a patient is presented in Fig. 2a, showing chronotropic response. The overall relationship between $\mathrm{HR}$ and all physical activities over the 24-h time window in all patients is presented in Fig. 2b, in which HR is significantly correlated with VM levels of physical activities. The correlation coefficients were at the same level between non-CI patients (Fig. 2c) and CI patients (Fig. 2d). However, the frequency of HR over $100 \mathrm{bpm}$ during activity events was significantly higher $(P=0.0006)$ in non-CI patients $(20 \%$ of all activity events, Fig. 2 c) than in CI patients $(8.8 \%$ of all activity events, Fig. $2 \mathrm{~d})$.

Maximal HR achieved during daily physical activities was $106.7 \pm 11.8 \mathrm{bpm}$ (equal to $63.9 \pm 6.8 \%$ APMHR) in CI patients, which was lower than $121.8 \pm 17.3 \mathrm{bpm}$ $(73.0 \pm 9.6 \%$ APMHR $)$ in non-CI patients $(P=0.020$ vs. CI patients). The maximal HR reserve achieved during the 24-h window was $31.9 \pm 7.0 \mathrm{bpm}$ in CI patients, significantly smaller than that in non-CI patients (40.9 $\pm 12.2 \mathrm{bpm} P=0.035$ vs. CI patients). The overall intensity (VM) of daily maximal physical activities was $333.2 \pm 87.6$ counts in CI patients, which was smaller than in non-CI patients $(390.0 \pm 118.8$ counts, $P=0.178$ vs. CI patients).
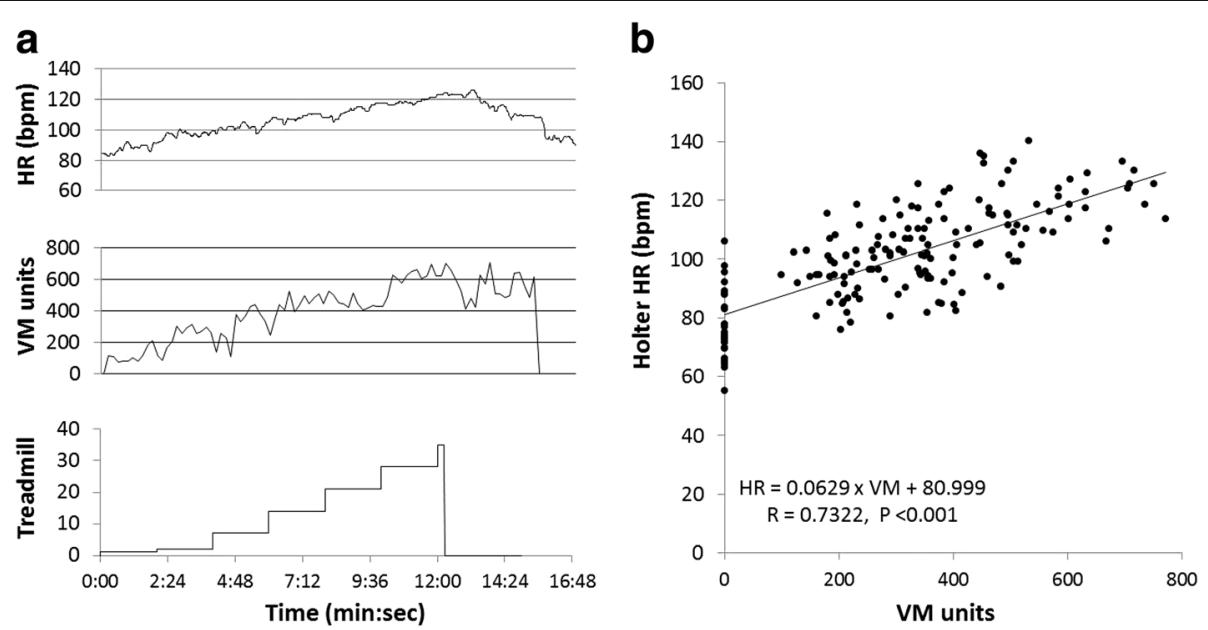

Fig. 1 Heart rate and Actigraph VM recording during treadmill exercise testing. a Holter heart rate (top) and Actigraph activity in VM (middle) recording during 7 levels of treadmill exercise testing (bottom) in one patient. $\mathbf{b}$ the correlation between Holter heart rate and levels of VM during treadmill exercise testing in all 26 patients. Each dot represents the measurement of a maximal VM value in one of 7 levels of treadmill exercise testing 

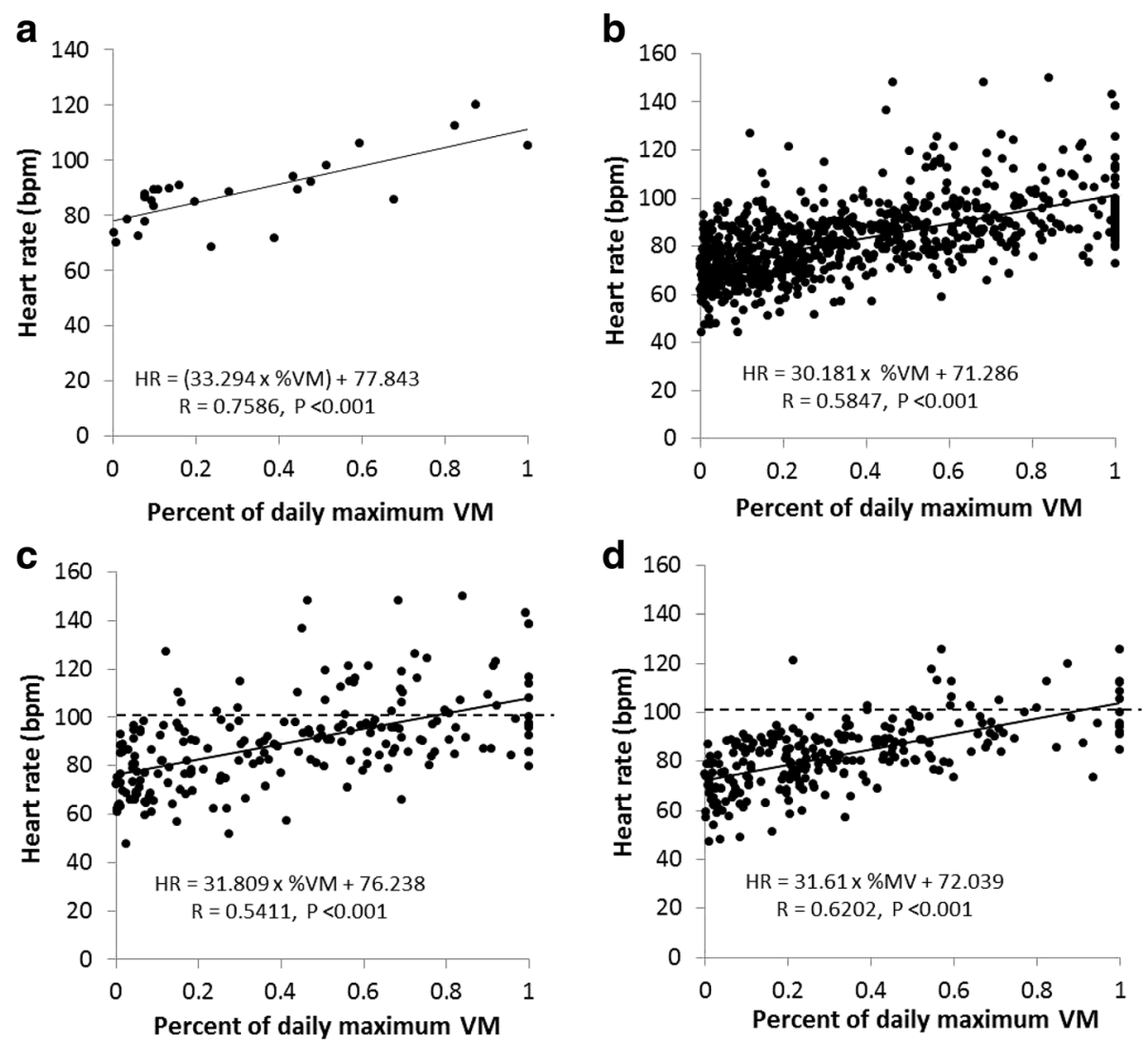

Fig. 2 Correlation between heart rate and physical activities during daily living. a the correlation between heart rate (bpm, the ordinate) and the percentage of each maximal daily physical activity over the greatest maximal daily physical activity (the abscissa) in one patient; $\mathbf{b}$ the correlation for all 40 patients; c the correlation for 13 non-Cl patients; $\mathbf{d}$ the correlation for $13 \mathrm{Cl}$ patients. Dashed line in (c and $\mathbf{d}$ ) represents the maximal HR above 100 bpm during physical activity events

HR analysis during 6-min hall walk

Maximal HR achieved during 6MHW (100.1 \pm 19.6 bpm) was significantly lower than maximal HR achieved during the peak of treadmill exercise testing $(112.0 \pm 14.8 \mathrm{bpm}$, $P=0.012$ ). However, there was a significant correlation between the $6 \mathrm{MHW}$ distance and the corresponding percent APMHR achieved during treadmill exercise testing $($ Distance $=571.7 \times$ \%APMHR $+68.6, R=0.615$, $P=0.001)$. The percent APMHR achieved during $6 \mathrm{MHW}$ was $57.5 \pm 6.3 \%$ in CI patients which was significantly smaller than that in non-CI patients (63.5 $\pm 6.9 \%$, $P=0.038$ vs. CI patients) and the $6 \mathrm{MHW}$ distance was significantly shorter in CI patients $(416.7 \pm 37.1 \mathrm{~m})$ than in non-CI patients $(486.2 \pm 62.0 \mathrm{~m}, P=0.003$, Table 1$)$.

\section{$\mathrm{Cl}$ assessment in patients without treadmill exercise testing}

Fourteen patients did not take treadmill exercise testing based on patients' willingness and physicians' clinical judgment. In these 14 patients, the achieved percent APMHR was $60 \pm 6.3 \%$ during daily physical activities and $56.4 \pm 7.9 \%$ during $6 \mathrm{MHW}$, both values of the percent APMHR were significantly lower than in non-CI patients $(73.0 \pm 9.6 \%$ APMHR during daily activities and $63.5 \pm 6.9 \%$ during $6 \mathrm{MHW}$, both $P$ values $<0.05$ vs. corresponding values in 14 patients), but comparable to the values of the percent APMHR in CI patients (63.9 $\pm 6.8 \%$ APMHR during daily activities and $57.5 \pm 6.3 \%$ during $6 \mathrm{MHW}$, both $P$ values $>0.05$ vs. the corresponding values in 14 patients). Moreover, the overall intensity (VM) of daily maximal physical activities was $315.5 \pm 107.8$ counts in VM, which appeared lower than in non-CI patients $(390.0 \pm 118.8$ counts, $P=0.101)$ but not significantly different compared with CI patients (333.2 \pm 87.6 counts, $P>0.05)$.

\section{Discussion}

The present study used two methods to assess impaired chronotropic response to physical activities in patients with heart failure. The method of traditional treadmill exercise testing identified $50 \%$ of patients whose maximal HR during peak exercise failed to reach at least $70 \%$ of APMHR, a criterion for CI diagnosis in heart failure patients $[12,19,20]$. Holter-Actigraph recording 
and corresponding measurements showed a high concordance with the results of exercise testing. Furthermore, in the 24-h recording of HR and physical activities by the Holter-Actigraph system, the assessment of chronotropic response during 6-MHW and daily living found worse chronotropic response in CI patients than in non-CI patients, demonstrating the feasibility of assessment of impaired chronotropic response in heart failure patients by this method.

In the present study, maximal HR during peak exercise failed to reach at least 70\% of APMHR in 50\% of tested patients, thus CI was diagnosed accordingly for these patients. When HR reserve and chronotropic index- $\beta$ were used to assess CI, 21 (80.8\%) of 26 patients who took exercise testing failed to attain $\geq 80 \%$ of HR reserve and $10(38.5 \%)$ patients failed to attain $\geq 60 \%$ of the chronotropic index- $\beta$. Thus, the CI incidence in heart failure population depends on the methods used for diagnosis [8]. The criterion of 70\% APMHR was used in the present study, based on which the study found that (1) patients who met the CI diagnosis criterion had a significantly lower HR reserve and chronotropic index- $\beta$ than those who did not meet the CI diagnosis criterion, and (2) HR changes in response to daily physical activities and the $6 \mathrm{MHW}$ distance were significantly smaller in patients who met the CI criterion than those who did not. When compared to the general population who have no heart failure and can reach the target heart rate during exercise testing (e.g., $\geq 85 \%$ APMHR) $[7,8]$, the heart failure patients in the present study whose heart rate during exercise testing could reach $\geq 70 \%$ APMHR, but still less than $85 \%$ might have reduced chronotropic response even though the $\mathrm{CI}$ could not be definitively diagnosed.

The present study applied a wearable recording system (Holter-Actigraph) during treadmill exercise testing in heart failure patients and assessed the correlation between treadmill exercise test results and measurements by Holter-Actigraph recording. The rationale to adopt Holter-Actigraph recording was to validate an ease-ofuse tool that can be used to screen for and assess CI without the need of special infrastructure like a treadmill exercise test laboratory. The uniqueness of the present study was to use Holter-Actigraph recording to determine HR during physical activities during daily living including $6 \mathrm{MHW}$ and thus evaluate chronotropic capacity. The analysis of HR and physical activities based on Holter-Actigraph recording revealed a significant correlation between heart rate and physical activity levels, e.g., chronotropic response. Furthermore, the study found a significant correlation between the $6 \mathrm{MHW}$ distance and the percent APMHR achieved during exercise testing and reduced physical activity intensity in patients with impaired chronotropic incompetence. Thus, the Holter-
Actigraph system can potentially be used to screen for $\mathrm{CI}$ and assess impaired chronotropic response in heart failure patients.

Exercise intolerance and symptoms of dyspnea and fatigue on effort are clinical manifestations in patients with heart failure. It is assumed that the appearance of these symptoms is more likely due to heart failure if rapid heart rate occurs in response to a moderate activity rather than the cause of $\mathrm{CI}$ in which there is a lack of a significant increase in heart rate. Thus, heart rate changes in response to a physical activity can be used to distinguish whether symptoms are caused by CI or not.

\section{Clinical perspectives}

Recent studies have demonstrated a high incidence of CI in the heart failure population and $\mathrm{CI}$ has been recognized as an independent risk factor for cardiovascular morbidity and mortality. Daily exercise is recommended for patients with chronic heart failure. However, the exercise intensity in terms of magnitude and time interval still remains uncertain and the intensity of exercise is significantly influenced by the capability of chronotropic response. Furthermore, use of $\beta$-blockers complicates chronotropic response, leading to a reduced chronotropic response $[8,9,11,21-23]$. On the other hand, $\beta$-blockers have been a standard therapy that prolongs survival of heart failure patients in several clinical trials [24-26]. There are approximately 5.8 million patients with heart failure in the United States and 23 million worldwide [27]. It can be assumed that the majority of heart failure patients would not receive an assessment of their chronotropic response. As shown in the present study, of 40 heart failure patients, 14 (35\%) patients who declined treadmill exercise testing did have low \%APMHR achieved during 6MHW and daily physical activities. Thus, if a simple, ease-of-use tool can be used to screen for $\mathrm{CI}$ and assess impaired chronotropic response in heart failure population, more patients with impaired chronotropic response could be identified and better managed during heart failure treatment including exercise-based cardiac rehabilitation and treatment with $\beta$-blockers. Rate-responsive pacing is an effective method to relieve symptoms caused by CI $[8,28]$. However, such a therapy may be underutilized in the heart failure population because exercise intolerance-related symptoms are more often recognized as heart failure symptoms instead CI-caused symptoms. Exertional dyspnea and weaknessasthenia are common symptoms in both chronic heart failure and CI. If drug therapy, such as loop diuretics, does not effectively relieve exertion-related symptoms in heart failure patients with $\mathrm{CI}$, rate-responsive pacing can be considered. In addition, $\mathrm{CI}$ can be a manifestation of sick sinus dysfunction whose symptoms include dyspnea, asthenia, fatigue, frequent dizziness, and possible fainting 
or syncope with the potential of precipitating or aggravating a state of heart failure [29]. Thus, rate-responsive pacing can be used if these patients receive a pacemaker. The present study clinically not only confirmed a high CI incidence in heart failure patients, but also investigated the feasibility of using Holter-Actigraph recording as an alternate and simpler tool to identify heart failure patients with CI and impaired chronotropic response. Prospective studies are needed to evaluate clinical benefits that can be provided by the assessment of chronotropic impairment with the use of tools as the Holter-Actigraph recording.

\section{Limitations}

The present study used the symptom-limited maximal exercise test that did not have respiratory monitoring or measurement of peak oxygen consumption. Thus, the present study did not incorporate the information of metabolic and oxygen demand during exercise. Second, the present study had a relatively small sample size and enrolled heart failure patients with LVEF ranging from $35 \%$ to $53 \%$. Moreover, exercise intolerance is one of the clinical manifestations of heart failure and use of $\beta$-blockers further complicates chronotropic response $[8,11,21-23]$. Thus, the variables for determining CI or chronotropic impairment derived from the present study need to be further confirmed in a clinical study with a large population including patients with all levels of LVEF and different medications.

\section{Conclusions}

The present study found $50 \%$ of tested patients whose maximal HR during peak exercise failed to reach at least $70 \%$ of APMHR, a criterion for CI diagnosis in heart failure patients. Use of the HR-activity recording system identified worse chronotropic response and lower physical activity intensity in CI patients than in non-CI patients, demonstrating the feasibility of assessment of impaired chronotropic response in heart failure patients by this simple method. Clinical utility of non-invasive monitoring tools as the Holter-Actigraph system in diagnosing and treating CI should be investigated in a larger heart failure population.

\section{Abbreviations}

6MHW: 6 min hall walk; APMHR: Age-predicted maximal heart rate value; $\mathrm{Cl}$ : Chronotropic incompetence; ECG: Electrocardiography; HR: Heart rate; LVEF: Left ventricular ejection fraction; VM: Vector magnitude

\section{Acknowledgements}

The authors wish to thank Dr. Hongyang Lu, a scientist at Medtronic Shanghai Innovation Center, for his assistance in the discussions and revisions of the manuscript.

\section{Funding}

No funding was received.

\section{Availability of data and materials}

The corresponding data are available upon request from Dr. Li at jbli@medmail.com.cn.

\section{Authors' contributions}

All authors helped to draft the manuscript. In addition, $J \mathrm{~L}, \mathrm{JZ}$ and $\mathrm{XZ}$ contributed to conception, study design, result interpretation and manuscript writing. HS and HL contributed to data acquisition, analysis, and interpretation. QW, JH and LW contributed to result interpretation and revised manuscript. SY and PW contributed to data acquisition and analysis. All authors read and approved the final manuscript.

\section{Competing interests}

Xiaohong Zhou, Shungang Yang and Ping Wang are Medtronic employees. To the best of authors' knowledge, Medtronic holds no stocks or shares in NorthEast Monitoring Inc. or ActiGraph. Medtronic may use the content of this manuscript as a reference for patent applications.

\section{Consent for publication}

Not applicable.

\section{Ethics approval and consent to participate}

The study was conducted in two centers and the study protocol was approved by both hospitals' Institutional Review Boards (Shanghai Sixth People's Hospital: Approval 2014-43(1), Ruijin Hospital Luwan Branch: Approval 2014-1) and in compliance with the Declaration of Helsinki. All patients completed written informed consent.

\section{Publisher's Note}

Springer Nature remains neutral with regard to jurisdictional claims in published maps and institutional affiliations.

\section{Author details}

'Department of Cardiology, Shanghai Sixth People's Hospital, Shanghai Jiao Tong University, Shanghai, China. ${ }^{2}$ Department of Cardiology, Ruijin Hospital Luwan Branch, Shanghai Jiao Tong University School of Medicine, Shanghai, China. ${ }^{3}$ Cardiac Rhythm and Heart Failure, Research and Technology, Medtronic plc, Mounds View, MN, USA. ${ }^{4}$ Department of Cardiology, Ruijin Hospital, Shanghai Jiao Tong University School of Medicine, Shanghai, China. ${ }^{5}$ Medtronic Shanghai Innovation Center, Medtronic (Shanghai) Ltd., Shanghai, China.

Received: 9 February 2017 Accepted: 15 May 2017

Published online: 25 May 2017

References

1. http://www.heart.org/HEARTORG/HealthyLiving/PhysicalActivity/ FitnessBasics/American-Heart-Association-Recommendations-for-PhysicalActivity-in-Adults_UCM_307976_Article.jsp.

2. Achttien RJ, Staal JB, van der Voort S, et al. Exercise-based cardiac rehabilitation in patients with chronic heart failure: a Dutch practice guideline. Nath Heart J. 2015;23:6-17.

3. Sagar VA, Davies EJ, Briscoe S, et al. Exercise-based rehabilitation for heart failure: systematic review and meta-analysis. Open Heart. 2015;2:e000163.

4. Carvalho VO, Mezzani A. Aerobic exercise training intensity in patients with chronic heart failure: principles of assessment and prescription. Eur J Cardiovasc Prev Rehabil. 2011;18:5-14.

5. Camm AJ, Lu F. Chronotropic incompetence-clinical implications. Clin Cardiol. 1996;19:503-8.

6. Tanaka H, Monahan KD, Seals DR. Age-predicted maximal heart rate revisited. J Am Coll Cardiol. 2001;37:153-6.

7. Brubaker PH, Kitzman DW. Prevalence and management of chronotropic incompetence in heart failure. Current Cardiol Rep. 2007;9:229-35.

8. Brubaker PH, Kitzman DW. Chronotropic incompetence: causes, consequences, and management. Circulation. 2011;123:1010-20.

9. Khan MN, Pothier CE, Lauer MS. Chronotropic incompetence as a predictor of death among patients with normal electrograms taking beta blockers (Metoprolol or Atenolol). Am J Cardiol. 2005;96:1328-33.

10. Savonen KP, Kiviniemi V, Laukkanen JA, et al. Chronotropic incompetence and mortality in middle-aged men with known or suspected coronary heart disease. Eur Heart Journal. 2008;29:1896-902. 
11. Dobre D, Zannad F, Keteyian SJ, et al. Association between resting heart rate, chronotropic index, and long-term outcomes in patients with heart failure receiving b-blocker therapy: data from the HF-ACTION trial. Eur Heart Journal. 2013;34:2271-80

12. Magrì $\mathrm{D}$, Corrà U, Lenarda $A D$, et al. Cardiovascular mortality and chronotropic incompetence in systolic heart failure: the importance of a reappraisal of current cut-off criteria. Eur J of Heart Failure. 2014;16:201-9.

13. Clark AL, Coats AJS. Chronotropic incompetence in chronic heart failure. International J Cardiol. 1995:49:225-31.

14. Roche F, Pichot V, Da Costa A. Chronotropic incompetence response to exercise in congestive heart failure, relationship with autonomic status. Clin Physiol. 2001;21:335-42.

15. Silva DA, Lima AC, Vargas FT, et al. Association between physical activity measurements and key parameters of cardiopulmonary exercise testing in patients with heart failure. J Cardiac Fail. 2013;19:635-40.

16. Garcia-Ortiz L, Recio-Rodriguez Jl, Puig-Ribera A, et al. Blood pressure circadian pattern and physical exercise assessment by accelerometer and 7-day physical activity recall scale. Am J Hypertension. 2014;27:665-73.

17. García-Ortiz L, Recio-Rodríguez Jl, Schmidt-Trucksäss A, et al. Relationship between objectively measured physical activity and cardiovascular aging in the general population - the EVIDENT trial. Atherosclerosis. 2014;233:434-40.

18. Patterson JA, Naughton J, Pietras RJ, et al. Treadmill exercise in assessment of the functional capacity of patients with cardiac disease. Am J Cardiol. 1972:30:757-62.

19. Meyer TE, Day JD, Merkel S, et al. Predictors of Chronotropic incompetence in heart Failure patients. J Card Fail. 2006;12:S18. (abstr)

20. Martin DO, Day JD, Murphy A, et al. Does Chronotropic incompetence limit the functional status of heart failure patients? J Card Fail. 2008;14:S67. (Abstr)

21. Witte KKA, Cleland JGF, Clark AL. Chronic heart failure, chronotropic incompetence, and the effects of $\beta$ blockade. Heart. 2006;92:481-6.

22. Gauri AJ, Raxwal VK, Roux $L$, et al. Effects of chronotropic incompetence and $\beta$-blocker use on the exercise treadmill test in men. Am Heart J. 2001:142:136-41.

23. Magri D, Palermo P, Cauti FM, et al. Chronotropic Incompentence and functional capacity in chronic heart failure: no role of $\beta$-blockers and $\beta$ blocker dose. Cardiovasc Ther. 2012;30:100-8.

24. Packer M, Bristow MR, Cohn JN, et al. The effect of Carvedilol on morbidity and mortality in patients with chronic heart failure. N Engl J Med. 1996;334:1349-55.

25. The RESOLVD Investigators. Effects of Metoprolol CR in patients with ischemic and dilated Cardiomyopathy. Circulation. 2000;101:378-84.

26. MERIT-HF Study Group. Effect of metoprolol CR/XL in chronic heart failure: Metoprolol CR/XL randomised intervention trial in congestive heart failure (MERIT-HF). Lancet. 1999 Jun 12:353(9169):2001-7.

27. Bui AL, Horwich TB, Fonarow GC. Epidemiology and risk profile of heart failure. Nat Rev Cardiol. 2011:8:30-41.

28. Tse HF, Siu CW, Lee KLF, et al. The incremental benefit of rate-adaptive pacing on exercise performance during cardiac resynchronization therapy. J Am Coll Cardiol. 2005;46:2292-7.

29. Semelka M, Gera J. Sick sinus syndrome: a review. Am Fam Physician. 2013:87(10):691-6.

\section{Submit your next manuscript to BioMed Central and we will help you at every step:}

- We accept pre-submission inquiries

- Our selector tool helps you to find the most relevant journal

- We provide round the clock customer support

- Convenient online submission

- Thorough peer review

- Inclusion in PubMed and all major indexing services

- Maximum visibility for your research

Submit your manuscript at www.biomedcentral.com/submit

C Biomed Central 